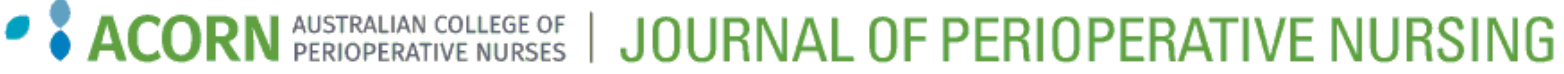

\title{
Asleep on the job: Can night shift napping provide greater safety for both staff and patients?
}

Follow this and additional works at: https://www.journal.acorn.org.au/jpn

Part of the Perioperative, Operating Room and Surgical Nursing Commons (c) (i)

This work is licensed under a Creative Commons Attribution 4.0 License.

\section{Recommended Citation}

Gamble, Jennifer and Foran, Paula (2021) "Asleep on the job: Can night shift napping provide greater safety for both staff and patients?," Journal of Perioperative Nursing: Vol. 34 : Iss. 1 , Article 6. Available at: https://doi.org/10.26550/2209-1092.1118

https://www.journal.acorn.org.au/jpn/vol34/iss1/6

This Article is brought to you for free and open access by Journal of Perioperative Nursing. It has been accepted for inclusion in Journal of Perioperative Nursing by an authorized editor of Journal of Perioperative Nursing. 


\title{
Authors \\ Jennifer Gamble \\ MCN (Perioperative Nursing), BN, BSC, \\ Grad Dip Nursing (Perioperative), FACPAN, MACORN \\ Asleep on the job: Can night shift napping provide greater safety for both staff and patients?
}

\begin{abstract}
Fatigue presents as an all-pervasive challenge to anyone who is engaged in shift work. While this affects all nurses, those who work on night duty have been shown to have the greatest risk of fatigue and cognitive impairment thus potentially posing a higher safety risk to themselves, the patients under their care and the wider community as nurses make their way home driving while drowsy.
\end{abstract}

Despite robust evidence revealing the positive attributes of increased working abilities from napping on night duty, and the overall agreement by nurse managers and organisations, this practice has been slow to be introduced into nursing. Research revealed that 55 per cent of nurse managers felt the greatest barrier to implementation was the lack of policy and supervision around this practice.

This discussion paper challenges nurses, managers and organisations to take note of current research, ACORN guidelines and work health and safety guidelines and start to change thinking and culture by looking at napping on night duty as a 'safety' implementation for staff, patients and the wider community. Policies and procedures should be developed and further research should be conducted into length and type of naps and subsequent positive and/or negative effects that may be reported by staff and health care facilities.

Keywords: napping, fatigue, night-duty

\section{Introduction}

All major hospitals must provide access to surgical and procedural services 24 hours a day; therefore, perioperative nursing is an area impacted by fatigue and staff-related sleep disturbances'. While fatigue affects all nurses, those who work on night duty have been shown to have the greatest risk of cognitive impairment, thus potentially posing a risk to both their own and patient safety ${ }^{2}$. Night duty napping is one technique that has been identified to help counteract this ${ }^{3,4}$. This discussion paper will examine if the practice of napping might be beneficial in terms of staff and patient safety, why there has been limited uptake of this practice and what barriers may exist to its uptake. This will be investigated under the following five themes: nurse fatigue on night duty, medico-legal considerations and implications, driving after night duty, research into napping at work and barriers to night shift napping.

\section{Nurse fatigue on night duty}

Nurse fatigue is a worldwide problem contributing to decline in the health of staff, errors in patient care and absence from work due to sickness ${ }^{2}$. Shift work has been shown to be a contributing factor in causing fatigue for all perioperative nurses ${ }^{5}$; however, the additional risk of working night duty is currently being 
examined $^{2}$. The Australian College of Perioperative Nurses (ACORN) guideline 'Fatigue' suggests that fatigue is an issue that has plagued perioperative nursing due to an eight- to ten-hour working day, the common need to work overtime and on-call shifts ${ }^{5}$. ACORN identified the significant health and safety risks that fatigued staff present, not only to themselves but also to their patients ${ }^{5}$.

A number of recent studies have clearly identified risks from nurse fatigue to staff, patients and the greater community². One observational study $(\mathrm{N}=90)$ conducted in Washington examined the impact on nursing staff comparing day and night shift workers working 12 hour shifts². Findings revealed that when night duty nurses reached the eight-hour mark of their shift, their cognitive performance was congruent with that of someone with a blood alcohol concentration of 0.05 per cent ${ }^{2}$. At the ten-hour mark their cognitive performance was congruent with that of someone with a blood alcohol equivalence of 0.08 per cent ${ }^{2}$. These results were only seen in the night duty cohort and not replicated in day shift participants ${ }^{2}$. Not surprisingly, findings also showed a statistically significant difference $(p<.001)$ in cognitive effectiveness where the night shift nurses experienced frequent and substantial decline to their cognition, compared to their day duty counterparts who did not ${ }^{2}$. These findings reveal safety concerns for both night duty nurses and their patients ${ }^{2}$.

\section{Medico-legal considerations and implications}

There are legal implications for nurses if fatigue affects their capacity to work safely. The Nursing and
Midwifery Board of Australia (NMBA) Code of Conduct - Principle 7 states that nurses must be mentally and physically fit to undertake their duties $^{6}$. Subsection (b) specifically states that nurses must ensure that fatigue does not impact on their practice $^{6}$. Failure to work outside the code of conduct can have serious implications for the registration of nursing staff ${ }^{6}$. ACORN included in their guideline on fatigue that nurses have an obligation to not be impacted by lack of sleep ${ }^{5}$. Although not legally binding, the ACORN standards and guidelines are the expected standards for practice for perioperative nurses in Australia ${ }^{5}$.

Safe Work Australia ${ }^{8}$ and Work Safe Victoria' both identify night duty as a high risk for fatigue and state that employers should try to roster to reduce the impacts of night duty and employees have the responsibility to divulge their fatigue. When viewing Standards for Perioperative Nurses in Australia (the ACORN Standards) with regards to fatigue ${ }^{5}$ in combination with work health and safety laws it is clear that perioperative nurses have a legal and professional responsibility to ensure they are not fatigued in order to provide safe care for their patients.

\section{Driving after night duty}

Salient research from 2007 reported that 79 per cent of night duty nursing staff stated that they felt drowsy when driving home from work? Despite this knowledge, driving home when feeling drowsy has continued to be a concern even with an elevated incidence of motor vehicle accidents and deaths being recorded in nursing night duty workers ${ }^{8}$. In a more recent study, conducted with intensive care unit nurses from Melbourne's Austin Hospital, participants ( $\mathrm{N}=33$ ) were asked to complete logs about their driving home from night shift in addition to wearing oculography glasses, devices that measure eyelid movement ${ }^{9}$. A key finding from that study was that nurses driving home were spending 4.4 per cent of each minute with their eyes closed, which at $100 \mathrm{~km} / \mathrm{hr}$ would result in travelling 74 metres with their eyes shut, for every minute of their commute?

Currently in Australia there is no single specific law that exists against driving a car or riding a motorbike while fatigued. However, approximately 20 to 30 per cent of all motor vehicle collisions in Australia are sleep-related, revealing that drowsiness is a leading cause of preventable road accidents and deaths ${ }^{10}$. Also, if found to be driving in a fashion that is considered unsafe, and given that fatigue is a condition that contributes to unsafe driving, there is the potential for drivers to be fined and given demerit points for careless or dangerous driving ${ }^{11}$. While all states and territories have their own set of driving regulations, the risk presented by fatigued drivers, in particular shift workers, would be the same and is highlighted by VicRoads on their website which states the onus is on employers to ensure employee fatigue is managed safely under work health and safety guidelines ${ }^{11}$.

\section{Research into napping at work}

While napping on night duty has been implemented in many safetysensitive industries as an effective countermeasure to drowsiness and fatigue, nursing has been slow to consider this option ${ }^{3}$. More recently, napping on night duty has been investigated to try and assess what length of nap provides best outcomes for improving cognition and lessening fatigue?

Hilditch et al. suggest that night shift napping should take place over 
ten minutes, as longer naps of 30 minutes or more may cause sleep inertia, the phenomenon of feeling confused, disoriented and slow upon waking ${ }^{4}$. Unlike most other research available on nursing night duty naps, Hilditch's study was conducted under laboratory conditions and the findings have not been replicated in real-world studies ${ }^{4}$.

In contrast, Geiger-Brown et al. found in their research that nurses only reported sleep inertia 1.3 per cent of the time, but that 56.2 per cent reported the nap left them feeling energised $^{11}$. Staff slept for 30 minutes and had a provision of 5 minutes either side of the scheduled sleep time to settle in and then to rouse and get moving again ${ }^{11}$. Participants in this study also reported feeling safer and more awake when driving home ${ }^{11}$. Following this study, other nurse unit managers contacted the authors with the intention to implement the model in their units because they witnessed the success of the project ${ }^{11}$.

In a study of South Australian nurses ( $\mathrm{N}=130)$ participants napped during their 45-minute meal break on night duty and reported needing less sleep during the day as well as improved ability to sleep ${ }^{12}$. Sleep inertia was not reported as an issue for these nurses.

The American College of Occupational and Environmental Medicine ran a task force on fatigue and produced guidance statements for organisations about how to limit fatigue for employees ${ }^{13}$. They identified napping for 30 minutes as an effective tool for employee work safety ${ }^{13}$. These sentiments were echoed by a recent, high-quality, evidence-based systematic review and meta-analysis by Dutheil et al. ${ }^{14}$. Their key findings indicated that the main cognitive function hampered when staff wake from a nap on night shift was memory, and that other cognitive processes were improved after napping ${ }^{14}$. This research counters the arguments that sleeping on shift means staff are compromised when woken to return to duty. Dutheil et al. also recommend that staff nap at an earlier time in their shift as opposed to 4.00 am or later ${ }^{14}$.

In regards to staff health, Li et al. conducted a mixed-methods systematic review of 22 studies and found that while napping was not conclusively proven to solve fatigue, there were definite health benefits related to it with regard to cardiovascular, metabolic and cognitive health, and reduction in muscular pain ${ }^{15}$. Their recommendation was for managers and staff to work together to find ways to implement napping for staff wellbeing ${ }^{15}$.

While napping is only one weapon in the arsenal against night duty fatigue, based on the high-quality evidence available there are robust grounds to support the concept that naps of around 30 minutes are an appropriate and effective safety intervention for night duty nurses.

\section{Barriers to night-shift napping}

Despite robust evidence revealing increased working abilities from napping on night duty $4,12,14,15$, this practice has been slow to be introduced into nursing 3 . In their cross-sectional survey of nurse managers $(\mathrm{N}=129)$, Dalky et al. found that 76.7 per cent of managers felt that there was benefit in staff having the opportunity to nap, and 55 per cent also knew of adverse patient and staff events that had been directly related to fatigue ${ }^{16}$. However, 63.6 per cent also felt that barriers, such as the lack of staff to cover the floor, increased workload for those not napping, the lack of appropriate space for napping and concern that the organisation did not support the activity were currently preventing implementation ${ }^{16}$. It was also noted that 55 per cent of nurse managers felt the greatest barrier to implementation was the lack of policy and supervision around this practice $^{16}$. Although not Australian research, this Jordanian study does give credence to the idea that nurse managers are supportive of staff having the opportunity to nap on shift.

Nurse managers and organisational policies, or lack thereof, have been identified as key challenges to staff napping overnight and it will only be through a change in culture, thinking and policy that staff will feel it appropriate and acceptable to nap on shift 1,3,13,15,16. Until organisations can understand and fully accept this practice as a safety initiative for both staff and patients, staff will have little choice but to 'sneak' a nap, or push through their fatigue, possibly causing risk to themselves and their patients.

Another barrier to implementation discussed in the literature is not having somewhere appropriate to sleep, such as a quiet, dark, comfortable area that is near colleagues in case the nurse who is napping is needed 1,3,12-14,16. Interestingly, the operating suite may be the one place in a hospital that has this facility as a trolley in an anaesthetic room would be suitable and nurses could bring their own sleeping sheet. Every perioperative suite has the appropriate facilities, but what is lacking is the governance and policy to allow nurses to use this as a safety initiative. Obviously, this would not be possible on very busy nights; however, in quiet periods this practice would allow staff to have greater cognitive function in preparation for the next case. 


\section{Conclusion}

This discussion paper has examined napping on night duty looking at nurse fatigue on night duty, medicolegal considerations and implications, driving after night duty, research into napping at work and barriers to night shift napping.

Evidence indicates that there are strong arguments to support perioperative nurses napping while on night duty as a matter of safety for themselves, the patients under their care and also for the wider community as nurses drive home from work. Barriers to implementation from managers and organisational culture have been identified and challenged. This debate must continue as providing a safe workplace is the shared responsibility of both the employers and employees. Policy development in this area is now vital to legitimise this practice.

It is also recommended that, as progressive hospitals begin this implementation, research be undertaken into length and type of naps as well as subsequent positive and/or negative effects that may be reported by staff and the health care facility.

Safety is at the core of perioperative nursing practice thus the caring and wellbeing of colleagues and patients must always be the first and foremost consideration of any perioperative nurse, no matter what shift they are on.

\section{Acknowledgment}

This paper was submitted to the University of Tasmania as part fulfilment of subject CNA803, Advanced Clinical Nursing Practice, for the Master of Clinical Nursing (Perioperative Nursing). The author sincerely wishes to thank Dr Paula Foran, unit coordinator, for her guidance throughout the master's course and work in preparing this paper for publication.

\section{References}

1. Querstret D, O'Brien, K, Skene D, Mabe J. Improving fatigue risk management in healthcare: A systematic scoping review of sleep-related/fatigue-management interventions for nurses and midwives. Int J Nurs Stud 2020;103:1-16.

2. James L, James $S$, Wilson M, Brown N, Dotson E, Edwards C. Sleep health and predicted cognitive effectiveness of nurses working 12-hour shifts: An observational study. Int J Nurs Stud 2020;112.

3. Geiger-Brown J, Sagherian K, Zhu S, Wieroniey M, Blair L, Warren J. Napping on the night shift: A two-hospital implementation project. Am J Nurs 2016;116(5):26-34

4. Hilditch C, Centofanti S, Dorrian J, Banks S. A 30-minute, but not a 10-minute night-time nap is associated with sleep inertia. Sleep 2016:39(3):675-684.

5. Australian College of Perioperative Nurses (ACORN). Standards for Perioperative Nursing in Australia, $16^{\text {th }}$ ed. Adelaide: ACORN; 2020

6. Nursing and Midwifery Board of Australia (NMBA). Code of Conduct for Nurses [Internet]. Canberra: NMBA: 2018 [cited 2020 September 27]. Available from: www. nursingmidwiferyboard.gov.au/CodesGuidelines-Statements/Professionalstandards.aspx.
7. Scott L, Hwang W, Rogers A, Nysse T, Dean G, Dinges $D$. The relationship between nurse work schedules, sleep duration and drowsy driving. Sleep 2007;30:1801-1807.

8. Smith A, McDonald A, Sasangoha F. Night-shift nurses and drowsy driving: $A$ qualitative study. Int J Nurs Stud 2020(112).

9. Mulhall M, Sletten T, Magee M, Stone J, Ganesan S, Collins A. Sleepiness and driving events in shift workers: The impact of circadian and homeostatic factors. Sleep 2019;42(6):1-13.

10. Austroads. Annual report 2016 [Internet]. Sydney: Austroads; 2016 [cited 2021 February 13]. Available from: www.austroads.com.au/ publications/corporate-reports-and-plans/ ap-c20-16.

11. VicRoads. Demerit point offences [Internet]. Melbourne: Department of Transport; 2019 [cited 2020 September 27]. Available from: www.vicroads.vic.gov.au/licences/demeritpoints-and-offences/demerit-offences.

12. Centofanti S, Banks S, Colella A, Dingle C, Devine L, Galindo H. Coping with shift work-related circadian disruption: A mixed-methods case study on napping and caffeine use in Australian nurses and midwives. Chronobiology Int 2018;35(6):853-864.

13. Lerman S, Eskin E, Flower D, George E, Gerson B, Hartenbaum N. The American College of Occupational and Environmental Medicine Guidance Statement: Fatigue Risk Management in the Workplace. J Occ \& Env Med. 2012;54(2):231-258.

14. Dutheil F, Bessonnat B, Pereira B, Baker J, Moustafa F, Fantin iM. Napping and cognitive performance during night shifts: A systematic review and meta-analysis. Sleep 2020;June.

15. L I H, Shao Y, Xing Z, Li Y, Wang S, Zhang M. Napping on night-shifts among nursing staff: A mixed-methods systematic review. J Adv Nurs 2019;75(2):291-312.

16. Dalky H, Raeda A, Esraa A. Nurse managers' perception of night-shift napping: A cross-sectional survey. Nurs Forum 2018;53(2):173-8. 